\title{
Fernsehen - Nutzen oder Schaden für die Familie?
}

\author{
von Ursula Lebr
}

\section{Einführung}

Das Medium Fernsehen wird für die Lebenswirklichkeit von Kindern und Eltern immer noch falsch eingeschätzt. Von den einen wird das Fernsehen geradezu verteufelt und als entwicklungsstörend betrachtet, als Schaden für die Entwicklung der Kinder und für das Familienleben. Von anderen wiederum wird das Fernsehen hochgelobt und als willkommener Miterzieher, als Bildungs- und Wissensvermittler betrachtet. Beide Einstellungen sind undifferenziert. Denn es gilt auch hier, nach dem rechten Maß zu suchen und differenziert zu fragen: für wen ist in welcher Situation welche Sendung von Nutzen und für wen ist in welcher Situation welche Sendung eher von Schaden?

\section{Fernsehen und Familie}

Das Fernsehen wirkt heutzutage in jede Familie hinein, gliedert sogar den Tagesablauf, bestimmt nicht selten die Essenszeiten, hat oft Einfluß auf familiäre Unternehmungen, auf Besuchszeiten und Besuchseinladungen. Der Bildschirm gehört längst zum selbstverständlichen Inventar unserer Wohnzimmer. Das Fernsehen sorgt für Informationen, bringt Orientierung über das Tagesgeschehen, sorgt für Bildung und Unterhaltung. Das Fernsehen bringt nun einmal die nahe und ferne Welt in die Familie hinein, bringt Gesprächsstoff und kann manche interessante Unterhaltung zwischen den Familienmitgliedern anregen. Freilich, es kann auch Unterhaltungen verhindern, kann auch Gespräche im Keim ersticken lassen. Aber das Fernsehen - vor allem durch ganz bestimmte Serien - prägt auch ganz entscheidend unser Bild von der Familie, die nicht immer realitätsorientiert dargestellt und oft sogar als Wert infrage gestellt wird leider! Hier bedarf es des Gesprächs im Familienkreis, der Aufarbeitung und Verarbeitung des Dargestellten.

Das Fernsehen ist aber für manch eine Familie auch zu einem Erziehungsmittel geworden. „Fernsehverbote" als Strafe oder die Erlaubnis zum Sehen bestimmter Sendungen als Belohnung für gute Noten sind keine Seltenheit. Doch auch als Betreuungsinstrument wird häufig das Fernsehen eingesetzt oder mißbraucht, damit Kinder beschäftigt sind bei zeitweiser Abwesenheit der Eltern. ${ }^{2}$ Andere Eltern lehnen es grundsätzlich ab und klagen darüber, welchen schlechten Einfluß das Fernsehen auf Kinder ausübe. Alle diese Verhaltensweisen verraten eine gewisse Unsicherheit: Weder schroffe Ablehnung noch uneingeschränktes Vertrauen sind angebracht.

Prof. Dr. Dr. h. c. Ursula Lehr war bis Januar 1991 Bundesministerin für Jugend, Familie, Frauen und Gesundheit und kehrt zum Sommersemester 1991 auf ihren Lehrstuhl für Gerontologie an der Universität Heidelberg zurück. 
Eine Welt ohne Fernsehen ist heute für uns nicht mehr denkbar. Aus einer Untersuchung aus der Schweiz ${ }^{3}$ wissen wir, daß $90 \%$ der Haushalte ein Fernsehgerät besitzen, $10 \%$ der Jugendlichen ein eigenes Gerät haben; in $60 \%$ der Haushalte gibt es Kabel-TV mit mehr als zehn Programmen, in $25 \%$ der Haushalte steht ein Video-Gerät zur Verfügung. $50 \%$ der Kinder und $85 \%$ der Jugendlichen besitzen ein eigenes Radio. Tele-Spiele, Pay-TV, Walkman, Kassettengeräte und Computer sind weit verbreitet. Das Medienangebot hat sich in den letzten Jahren erheblich ausgeweitet, und die Mediennutzung nimmt weiter zu. Mit der Ausbreitung der neuen Medien im Bereich der Unterhaltungselektronik hat sich die gesamte Freizeitsituation der Gesellschaft verändert. Veränderungen im Lebensstil sind nachweisbar, sowohl quantitativ durch das Ausmaß der hierfür aufgewendeten Zeit als auch durch qualitative Veränderungen im Familienleben.

Aus den Zahlen, die die Gesellschaft für Kommunikationsforschung (GfK) publiziert ${ }^{4}$, wird ersichtlich, daß die Fernsehnutzung 1988 bei über 14jährigen im Bundesdurchschnitt bei $21 / 2$ Stunden täglich, genauer bei 152 Minuten lag; in verkabelten Haushalten waren es sogar durchschnittlich $23 / 4$ Stunden, genau 164 Minuten (also 12 Minuten mehr) pro Tag. Doch man sollte dies nicht zum Anlaß nehmen, die Verkabelung zu verurteilen.

Mit der Einführung des Kabelfernsehens wurden eine Reihe von Untersuchungen angestellt, die speziell die Auswirkungen auf das Familienleben erfassen sollten. Ich selbst gehörte von 1980 bis 1987 der vom Land RheinlandPfalz einberufenen wissenschaftlichen Begleitkommission zum Versuch mit Breitbandkabeln an. Unsere sehr gründlichen Längsschnitterhebungen, in denen über 1.200 Familien in der Zeit von 1984 bis 1986 mehrmals erfaßt worden sind, zwingen jedoch zu sehr differenzierter Betrachtung hinsichtlich möglicher Auswirkungen des Fernsehens. ${ }^{5}$

Zunächst einmal ist festzustellen, daß das Fernsehen für die meisten Menschen das Medium ist, aus dem sie zuerst Neuigkeiten über das Weltgeschehen erfahren. Fernsehen als Informationsvermittler ist besonders geeignet, da es visuell und akustisch, also durch Bild und Ton wirkt. Interessant ist die Feststellung, daß die „verkabelten“ Untersuchungsteilnehmer „keineswegs als passive Freizeitgestalter einzustufen sind. Im Gegenteil: unter ihnen ist der Anteil, der während seiner Freizeit lieber aktiv ist, etwas unternimmt, größer als unter den Nichtteilnehmern". Sozialkontakte, aktives Sporttreiben, Theater- und Museenbesuche, Besuch von Weiterbildungskursen kommt sogar bei den „Vielsehern" häufiger vor. ${ }^{6}$

\section{Fernsehen und Kinder}

Über den Einfluß des Fernsehens auf die Entwicklung der Kinder ist viel geschrieben, gesprochen, diskutiert worden. Die Ergebnisse der Untersuchungen wie auch die Expertenfeststellungen sind äußerst widersprüchlich und reichen von eindeutigen Verurteilungen wie "das Fernsehen schadet Kindern", "das Fernsehen macht Kinder krank" ${ }^{\text {"7 }}$ bis hin zu Feststellungen, denen zufolge das Fernsehen das potentielle Verhaltensrepertoire der Kinder erweitern kön- 
ne ${ }^{8}$, oder - zumindest bei bestimmten Sendungen wie "Sesamstraße“, ,Rappelkiste“, "Lach- und Sachgeschichte" - eine Initiierung und Ausdehnung einer formalen Leistungsbereitschaft der Kinder bewirke; diese Sendungen seien über weite Strecken eine Art „Trimmt-Euch-Kurs“ zur Entwicklung der Formalintelligenz ${ }^{9}$.

Untersuchungen $^{10}$ zum Ausmaß des Fernsehkonsums bei Kindern und Jugendlichen haben festgestellt, daß Kinder aus Familien mit niedrigem sozioökonomischen Status häufiger fernsehen als Kinder aus Familien mit höherem Sozialstatus, daß ebenso in städtischen Gegenden von Kindern und Jugendlichen mehr ferngesehen wird als in ländlichen Gegenden; daß Jungen mehr fernsehen als Mädchen (im Schnitt eine halbe Stunde mehr); daß Kinder im Alter zwischen 10 und 13 Jahren die fernsehintensivste Altersgruppe sind, und daß Kinder von Eltern, die häufig fernsehen, auch selbst häufiger vor dem Fernseher anzutreffen sind. Je schwächer die Schulleistung, desto ausgedehnter der Fernsehkonsum; die seitens der Lehrer als „wenig aktiv“ beurteilten Schüler ließen einen Mehrkonsum von etwa 30 Minuten erkennen. Diese Feststellungen beruhen jedoch lediglich auf Korrelationen, die man nicht im Sinne einer UrsacheFolge-Beziehung deuten kann. Ob also erhöhter Fernsehkonsum Kinder passiv macht oder ob passive Kinder häufiger vom Fernsehen Gebrauch machen oder ob gar ein dritter Faktor sowohl für die Passivität der Kinder als auch für deren vermehrten Fernsehkonsum verantwortlich zu machen ist, kann daraus nicht interpretiert werden.

Jonson stellt in einer Untersuchung ${ }^{11}$ von Kindern zwischen 6 und 12 Jahren fest, daß Kinder von Müttern mit mehr Bildung und höherem sozioökonomischen Status mehr Erfolg in der Schule und gleichzeitig weniger Interesse an Unterhaltungssendungen hatten als Kinder von Müttern mit niedrigerem sozioökonomischen Status, die sowohl schlechtere Schulleistungen erbrachten als auch längere Zeit vor dem Fernsehschirm saßen. Doch weniger das Ausmaß des Fernsehkonsums als die von den Eltern vermittelte Einstellung zum Fernsehen zeigte sich in dieser Studie als entscheidender Faktor im Zusammenhang mit dem schulischen Verhalten der Kinder. Kinder, die von ihren Eltern dazu angeregt wurden, altersgemäße Programme zu sehen, sich mit den Inhalten auseinanderzusetzen und sich diese gegebenenfalls anzueignen, also Kinder, die einen kritischen Umgang mit dem Fernsehen zeigten, waren besser in der Lage, mit schulischen Anforderungen umzugehen als Kinder, die von ihren Eltern aufgefordert wurden, Fernsehen als Mittel zur Entspannung und Unterhaltung zu nutzen.

Eine der ältesten empirischen Untersuchungen ist jene von Himmelweit et al., ${ }^{12}$ die in England 4.500 fernsehende Kinder erfaßte und ebenso Kinder aus einer Gegend, in der zu dieser Zeit noch keine TV-Empfangsmöglichkeiten gegeben waren. Dort wurden - dank der idealen Untersuchungsbedingungen Kinder vor Kontaktaufnahme mit dem Fernsehen untersucht und nach einem Jahr erneut getestet. Unter Anwendung der Methode der „Kontroll-Zwillinge“ (Übereinstimmung nach Geschlecht, Alter, Intelligenz, sozialem Status, Schulklasse) wurde die Entwicklung während eines Jahres von Fernseh- und Nichtfernsehkindern verglichen. Dabei zeigte sich - neben vielen Einzelergebnissen, 
daß in der Gruppe der Fernsehkinder mit der Höhe des Intelligenzquotienten die vor dem Fernsehschirm verbrachte Zeit abnahm; mit steigender allgemeiner Aktivität und Interessiertheit wie auch mit steigender Sozialkontaktstärke reduziert sich ebenso die tägliche Dauer des Fernsehens. Generell wurde eine verstärkte Zuwendung zum TV konstatiert, solange das Gerät neu war; nach ungefähr dreimonatiger Gewöhnung erlahmte das intensive Fernsehinteresse. Ein Vergleich zwischen Fernsehkindern und Nicht-Fernsehern im Hinblick auf Allgemeinwissen und kognitive Leistungen zeigte, daß in der Gruppe der jüngeren Kinder (die noch keinen Zugang zum Buch hatten) Fernsehkinder während des einjährigen Untersuchungszeitraumes einen Wissensvorsprung erzielten. Auch in der Gruppe der weniger intelligenten älteren Kinder zeigten Fernsehkinder ebenso einen Vorsprung gegenüber Nicht-Fernsehkindern, - doch in der Gruppe der älteren und der intelligenteren Kinder hatten Fernseh-Kinder eine schlechtere Entwicklung als Nicht-Fernseher - sie wiesen im kognitiven Bereich (allgemeines Wissen, Intelligenz, Informiertsein) schlechtere Leistungen auf als Nicht-Fernseher, die sich den Stoff anderweitig erarbeiteten.

Ähnliche Differenzierungen nach Intelligenz, Persönlichkeit, familiärer Situation zeigten sich auch im Hinblick auf Aggressionen, Werthaltungen, Freizeitinteressen, so daß die Autoren vor zu simplen Beziehungsannahmen warnten: Fernsehen ist an sich weder als Gefahr noch als Gewinn zu sehen; weder als Nutzen noch als Schaden. Fernsehen ist nicht grundsätzlich als gut oder schlecht zu beurteilen, sondern seine Wirkungen sind immer im Zusammenhang mit bestimmten Programminhalten, bestimmten Persönlichkeitsmerkmalen der Kinder (und ihrer Eltern) und bestimmten situativen Bedingungen zu analysieren.

Die bereits zu Beginn der 50er Jahre begonnene Diskussion um die Auswirkungen des Fernsehens auf die kindliche Entwicklung ist auch in den 80er Jahren noch nicht verstummt - im Gegenteil, sie wurde durch die Einführung des Kabelfernsehens eher noch verstärkt.

Dabei werden die bereits vor 30 Jahren getroffenen Feststellungen nur bestätigt: Ob ein Einfluß überhaupt gegeben ist, ob dieser Einfluß unmittelbar, kurzfristig oder gar langfristig Auswirkungen zeigt, „hängt nicht so sehr (aber auch!) von den Sendungen ab, die das Kind sieht, als von dem Kind selbst ${ }^{* 13}$ und seiner sozialen Umwelt, seiner Familie. Schramm ${ }^{14}$ ist aufgrund seiner Untersuchungen an 600 Kindern zu der Erkenntnis gelangt: Wichtiger als das, was das Fernsehen zum Kind bringt, ist das, was das Kind zum Fernsehen mitbringt. Es gilt also, mögliche Einflußgrößen auf das Kind in Abhängigkeit von dessen Persönlichkeitsstruktur, von Alter, von Intelligenz, Interessenlage wie auch in Abhängigkeit von seiner familiären Situation bzw. dem familiären Klima und sonstiger situativer Gegebenheiten - und der Art des Angebots - zu diskutieren.

Die Abhängigkeit der Wirkungen von der Persönlichkeit des Kindes wie auch von den übrigen Sozialisationsinstanzen Elternhaus, Schule, Peer-Group sollte bei derartigen Diskussionen stärker als bisher berücksichtigt werden. Dort, wo eine intensive Bindung des Kindes an das Elternhaus gegeben ist, werden negative Auswirkungen weniger beobachtet. So kommt Maccoby zu der 
Feststellung eines relativ geringen Einflusses des Fernsehens auf jene Kinder, die sich in der familiären Bezugsgruppe geborgen fühlen und für die dann deren Wert- und Verhaltensmuster ausschlaggebend sind. Ob gesehenes Verhalten übernommen bzw. nachgeahmt wird, hängt davon $a b$, ob das Kind überhaupt in eine ähnliche Situation kommt und „ob das beobachtete Verhalten im Einklang mit den moralischen Grundsätzen ist, die man ihm beigebracht hat, und ob die sittliche Erziehung wirksam genug gewesen ist, das Kind zu befähigen, einer zusätzlichen Versuchung zu widerstehen". Ähnlich betont Herzberg's, daß das Fernsehen als Sozialisationsfaktor nur dort an Gewicht gewinnt, wo es Gruppenbeziehungen unterläuft oder ersetzt, und dort, wo die Identifikationsmöglichkeiten des Kindes mit seinen Bezugspersonen gestört oder gar nicht gegeben sind. ${ }^{16}$

Das Ergebnis unserer Längsschnitt-Untersuchungen in Rheinland-Pfalz läßt sich im Hinblick auf das Erleben des Familienlebens wie folgt zusammenfassen ${ }^{17}$ : Die Selbstbeurteilung des Familienlebens zeigt bei den KabelfernsehTeilnehmern ein Erleben einer mehr positiven Entwicklung, vor allem einen Rückgang der Feststellungen, daß zuhause eine "gereizte Stimmung“ herrsche und man oft " verschiedener Ansicht" sei. Intensive Kontakte, häufige Unterhaltungen der Familienmitglieder miteinander scheinen dann gegeben, wenn die Eltern eine bessere Schulbildung haben und ein höheres Familieneinkommen zur Verfügung steht. Hier gibt es keine Unterschiede zwischen Familien mit und ohne Kabelanschluß.

Auch die erlebte Gemeinsamkeit in der Familie - erfaßt mit den Items "wir unternehmen viel gemeinsam" und "wir lachen viel zusammen, haben viel Spaß miteinander" läßt nur Trends erkennen, erlaubt aber keine eindeutigen Feststellungen. Ein signifikanter Rückgang gemeinsamer Unternehmungen findet sich bei den Eltern der 14- bis 18jährigen zwar sowohl in der Teilnehmergruppe als auch in der Gruppe der Nicht-Teilnehmer. Eine Veränderung der familiären Kontakte ist also primär durch das Heranwachsen der Kinder bestimmt; das Vorhandensein oder Fehlen eines Kabelanschlusses hat sekundäre Bedeutung.

Die persönliche Bedeutsamkeit der Familie hat bei Teilnehmern und Nicht-Teilnehmern ihren Schwerpunkt im „Gefühl, geliebt zu werden“, Geborgenheit und Verständnis zu finden ( Die Möglichkeit, mit anderen Familienmitgliedern anregende Gespräche zu führen, wird in der Teilnehmergruppe sogar stärker hervorgehoben als in der Gruppe der Nicht-Teilnehmer.

Es ist hier nicht der Ort, auf die Methodenproblematik der Erfassung der Fernsehwirkung einzugehen. Wir sollten jedoch vorsichtig sein sowohl mit einer generellen Verurteilung des Fernsehens als auch mit einer generellen Hervorhebung positiver Auswirkungen auf die Entwicklung des Kindes bzw. des Jugendlichen. 


\section{Ausblick}

Hier sind die Eltern gefordert. Sendungen müsssen besprochen werden, sollten miteinander diskutiert werden. Es darf nicht sein, daß ein Kind, das während der Sendung Fragen stellt, als störend empfunden und zurechtgewiesen wird. Eltern sollten darüber diskutieren, wie die Personen in den Filmen miteinander umgehen, wie sie sich bei Konflikten verhalten und welche allgemeine Einstellung zum Leben sie überhaupt verkörpern - und Eltern sollten klar und deutlich zu erkennen geben, welches Verhalten der Filmhelden sie gut finden und - vor allem - warum sie es gut finden, und welches Verhalten sie für nicht gut finden - und warum sie es ablehnen. So können die Eltern anhand konkreter Beispiele ihre Wertmaßstäbe vermitteln.

Das Fernsehen kann die familiäre Erziehung in mehrfacher Weise beeinflussen. Einmal greift es oft in die Organisation des Familienalltags ein. Es konfrontiert außerdem die Familie mit einer Fülle von Wissen und Informationen aus dem Alltag, dem Zeitgeschehen, der Politik, der Wissenschaft und der Werbung. Das Fernsehen vermittelt allen Familienmitgliedern ein breites Angebot von Bildern aus dem Familienalltag, der vielfach realitätsfern - entweder als zu idyllisch und manchmal auch idealisiert oder aber als brutal, aggressiv und abstoßend - dargestellt wird. Durch das Fernsehen lernen Kinder und Jugendliche die Erwachsenenwelt in ihrer ganzen Vielfalt - aber dennoch in ausgewählten Episoden - kennen. Hier haben Eltern zu korrigieren.

Positive und negative Auswirkungen des Fernsehens sind abhängig von den Erziehungsbedingungen in der Familie, von der Mitarbeit der Eltern, von der Persönlichkeit des Kindes, von der Gestaltung seines sonstigen Lebensraumes und schließlich ganz entscheidend von der Qualität des Programms. Die Wechselwirkungen zwischen all diesen Komponenten bestimmen den Einfluß des Fernsehens und seine prägenden Wirkungen. Sie entscheiden darüber, ob das Fernsehen zum Nutzen oder Schaden für das Kind ist; ob der Bildschirm zur Belastung wird und Unheil anrichtet oder zur Bereicherung führt und sogar Entwicklungshilfen bietet.

Kinderfernsehen ist nach wie vor beliebt. Aber die Moden wechseln hier schnell. Was bei den Kindern heute noch beliebt ist, dem weinen sie vielleicht morgen schon keine Träne mehr nach. Nur wenige Helden und Mythen aus dem Kinderprogramm haben sich auf Dauer durchgesetzt, sind bei Kindern und Jugendlichen aller Altersgruppen bekannt. So gilt es, immer erneut das Programm zu überprüfen, immer erneut nach geeigneten Sendungen zu suchen und ungeeignete Sendungen auszuschließen. 
Anmerkungen:

1 Rede anläßlich der Verleihung des Fernsehpreises der Zeitschrift "leben \& erziehen “ und des Familienbundes der Deutschen. Katholiken am 18. Oktober 1990 in Bonn. Die Zwischentitel sind von der Redaktion eingefügt worden.

2 U. Lehr/E. Minnemann, Fernsehen: Wirkungen im familiären Bereich, in: Abschlußbericht der wissenschaftlichen Begleitkommission (s. Anm. 5), 251-301, 251ff.

$3 \mathrm{H}$. Bonfadelli, Kinder, Jugendliche und Medien: Vom Aufwachsen in einer elektronischen Umwelt, in: Bundesministerium für Jugend, Familie, Frauen und Gesundheit (Hg.), 40 Jahre Bundesrepublik Deutschland. Zur Zukunft von Familie und Kindheit, Bonn 1989, 101ff.

4 Vgl. ebd.

5 Wissenschaftliche Begleitkommission zum Versuch mit Breitbandkabel in der Region Ludwigshafen/Vorderpfalz (Hg.), Abschlußbericht an die Landesregierung Rheinland-Pfalz, Band 5, Berlin/Offenbach 1987.

6 Vgl. F. Landwehrmann/A. Topfmeier/M. Jäckel, Mediennutzung und Medienwirkung, in: Abschlußbericht der wissenschaftlichen Begleitkommission, 175-299, 201.

7 M. Winn, Die Droge im Wohnzimmer, Hamburg 1979.

8 E. E. Maccoby, Die Wirkung des Fernsehens auf Kinder, in: W. Schramm (Hg.), Grundfragen der Kommunikationsforschung ${ }^{5}$ 1973, 141-155

9 H. Holzer, Kinder und Fernsehen - Materialien zu einem öffentlich rechtlichen Dressurakt, München 1974.

$10 \mathrm{Vgl}$. F. Stückrath/G. Schottmeyer, Fernsehen und Großstadtjugend, Braunschweig 1967.

11 A. Jonson, TV - a threat or a complement to school?, in: Educational and Psychological Interactions, No. 82 (1985).

12 H. T. Himmelweit/A. N. Oppenheim/P. Vince, Television and the child: an empirical study of the effect of vision on the young, New York 1958.

13 E. Hurlock, Die Entwicklung des Kindes, Weinheim 1970.

$14 \mathrm{~W}$. Schramm, Children and television. Television Information Office, Washington D. C. 1959; ders., Mass media and educational policy. 60th Yearbook, Soc. Stud. Educ. 1961, 203-229.

15 I. Herzberg, Medienpädagogik im Kindergarten, in: J. Barthelmes et al. (Hg.), Untersuchungen zur pädagogischen Wirkung von Hörfunk und Fernsehen - Massenmedien und vorschulische Erziehung, Bd. III., München 1979.

16 Vgl. auch Landesanstalt für Rundfunk NRW (Hg.), Medienerziehung im Kindergarten: Neue Herausforderungen durch private Programme?, LfR-Dokumentation Band 3, Düsseldorf 1990.

17 Vgl. U. Lehr/E. Minnemann, a. a. O., 284f. 


\section{SUMMARY}

Undifferentiated assessments about the effect of television on children and on family life have long been untenable. Nevertheless again and again one finds statements which eihter condemn television unreservedly or accept and welcome it thoughtlessly as the most important means of education. The author points out with the help of several experimental results that these aproaches are not appropriate to the interests of children and families. Television today has become an integral part of daily life. This does not mean that a child's development is determinded or completely controlled by television. Simplistic assumptions about the influence of television consumption on the socialization of children are unfounded. Fundamentally the assessment of television is neither good or bad. It is, however, particularly important that children's television viewing is accompanied by parents who are attentive and prepared to talk to their children. Possible positive or negative influences of television on children depend essentially on the educational conditions in the family and on the co-operation of the parents.

\section{RÉSUMÉ}

On ne peut plus accepter d'estimations arbitraires qui portent sur les effets que la télévision exerce sur les enfants et la vie de famille. Pourtant, on rencontre parfois des propos qui condamnent de manière globale la télévision ou qui l'acceptent sans réserve en tant que co-éducateur. L'auteur se fonde sur plusieurs recherches pour accentuer que ces estimations sont déplacées quant à l'intérêt des enfants et des familles. La télévision est aujourd'hui devenue un élément intégral de la vie quotidienne. Mais cela ne veut pas dire que ce soit cette institution qui influence avant tout ou même totalement le développement des enfants. Les suppositions simples sur l'effet que la télévision a sur la socialisation des enfants sont inexactes. La télévision en soi n'est ni bonne ni mauvaise. Ce qui importe avant tout, c'est que les enfants ne devraient pas être laissés seuls devant la télévision, au contraire, il faut des parents attentifs et communicatifs. Les conséquences négatives ou positives de la télévision sur les enfants dépendent essentiellement des conditions dans lesquelles se déroule l'éducation familiale et de la collaboration des parents.

\section{RESUMEN}

Actualmente son insostenibles las opiniones no diferenciadas sobre el efecto de la televisión sobre los niños y la vida familiar. Empero se dan todavía algunas manifestaciones que o condenan la televisión de forma general o la ven y la aceptan como un coeducador importante. La autora hace notar, en base a diferentes investigaciones, que estas posturas no son adecuadas si se considera el interés de los niños y de las familias. La televisión se ha transformado en un elemento integrante de la vida cotidiana. Todo lo cual no significa que los niños no sean influídos decisivamente o incluso exclusicamente por la televisión. Simples conjeturas sobre la influencia del consumo televisivo para la socialización del niño, no son acertadas. La televisión no puede ser considerada ni buena ni mala. Especialmente importante es, sin embargo, el acompañamiento activo que se hace al niño en su consumo televisivo por parte de padres atentos y dispuestos a hablar con él sobre éso. De tal forma que los posibles influjos positivos o negativos de la televisión sobre los niños depende esencialmente de las condiciones educativas en la familia y de la colaboración de los padres. 